\title{
Relationship Between the Structure of Methoxylated and Hydroxylated Flavones and Their Antiproliferative Activity in HL60 Cells
}

\author{
SATORU KAWAII ${ }^{1}$, YUYA ISHIKAWA ${ }^{1}$ and YUKO YOSHIZAWA ${ }^{2}$ \\ ${ }^{1}$ Laboratory of Bio-organic Chemistry, Tokyo Denki University, Saitama, Japan; \\ ${ }^{2}$ Laboratory of Bio-organic Chemistry, Akita Prefectural University, Akita, Japan
}

\begin{abstract}
As part of our continuing investigation on flavonoid derivatives as potential anticancer substances, a series of methoxylated and hydroxylated flavones was synthesized, and their cytotoxic and anti-proliferative activity was evaluated in leukemic HL60 cells. Their structureactivity relationship was also investigated. The correlation between the methoxylation/hydroxylation pattern and antiproliferative activity revealed the importance of the 5,4'and 3',4'-dihydroxyl moieties in flavone nucleus.
\end{abstract}

Many of the health-promoting effects of fruits, vegetables, legumes and beverages such as wine, coffee and tea, are attributed to flavonoids, which are naturally occurring polyphenolic compounds (1-4). They occupy an important part of human diet, and are reported to have a broad spectrum of biological activities, such as antibacterial and anti-inflammatory activities. They also participate in cellular mechanisms related to cancer. Although the positive biological actions of flavonoids have been assigned to their antioxidant properties (5), there is an emerging view that flavonoids and their metabolites do not act only as hydrogendonating antioxidants, but could also modulate signaling pathways in cells (6). Flavonoids might be used not only for cancer prevention (7), but also as anticancer agents $(8,9)$.

Polymethoxyflavones (PMFs) are flavones substituted with two or more methoxyl groups. PMFs are found almost exclusively in the Citrus genus particularly in the peel of king orange $(C$. nobilis), sweet orange $(C$. sinensis), and mandarin orange $(C$. reticulata). They are coming to the center of interest due to their documented wide spectrum of

Correspondence to: Professor Satoru Kawaii, Faculty of Science and Engineering, Tokyo Denki University, Hatoyama, Saitama, 3500394, Japan. Tel: +81 492965847, e-mail: kawaii@ mail.dendai.ac.jp

Key Words: Methoxyflavone, hydroxyflavone, antiproliferative activity, HL60 cells, structure and activity relationship. biological activity including anti-inflammatory, anticarcinogenic, anti-atherogenic, neurotropic and memoryenhancing properties (10-14). PMFs have also been reported to inhibit the growth of HL60 cells in vitro and to suppress proliferation, while promoting apoptosis (15). The potent differentiation-inducing activity toward HL60 leukemic cells was found from in the juice of Citrus nobilis, and the active components were isolated and identified as four PMFs (16). Their biological properties lead to horticultural breeding attempt to produce new citrus cultivar containing high amounts of PMFs $(17,18)$.

Our investigation on the structure-activity relationship of PMFs showed that there was a complicated correlation between the number/position of methoxyl group and their antiproliferative activity. Roughly speaking, an increase in the number of methoxyl groups on the A-ring enhanced the activity of PMFs, whereas the increase of B-ring methoxyl groups reduced the activity (19). In order to determine how the methoxyl/hydroxyl groups contribute to the antiproliferative activity of flavone, relatively simple methoxylated flavones and their corresponding hydroxylated flavones were synthesized and their antiproliferative activity was estimated.

\section{Materials and Methods}

General procedures. Chemicals and solvents from commercial sources were used without further purification unless specified. Reactions were carried out under argon, and monitored by thinlayer chromatography on silica gel (mesh size 60, F254) and with visualizedation under UV light. Standard and flash column chromatography procedures were not optimized. Nuclear magnetic resonance (NMR) spectra were recorded on a $400-\mathrm{MHz}$ JEOL ECP-400 spectrometer (JEOL, Tokyo, Japan), and chemical shifts values are expressed in $\mathrm{ppm}(\delta)$ relative to the residual ${ }^{1} \mathrm{H}$ signal of the solvents. Unless otherwise specified, compounds were dissolved in dimethylsufoxide- $\mathrm{d}_{6}$. Electrospray ionization (ESI) and atmospheric pressure chemical ionization (APCI) mass spectrometry were performed on Thermo Exactive (Thermo Fisher Scientific K.K, Tokyo, Japan) and Hitachi M8000 instruments (Hitachi, Tokyo, Japan), respectively. 


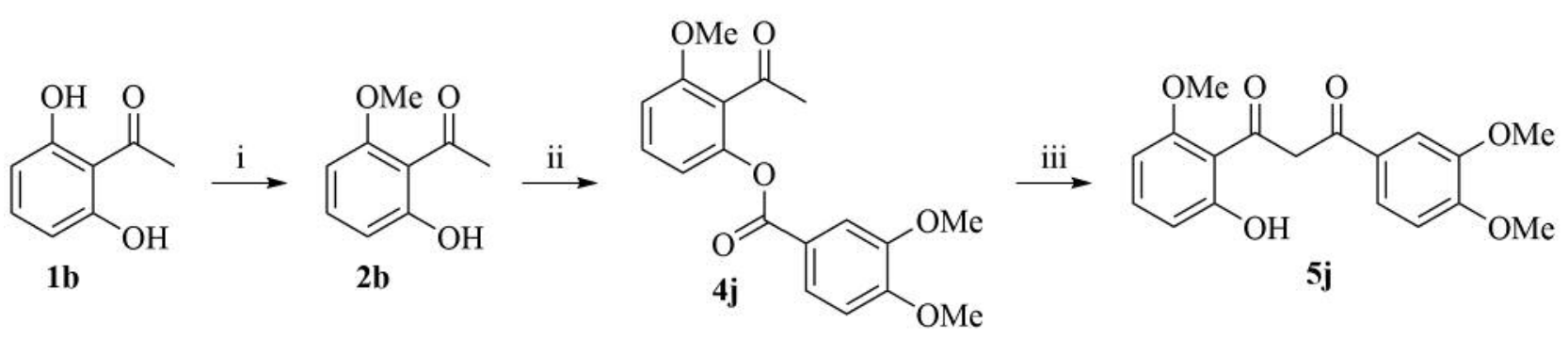<smiles>COc1ccc(-c2cc(=O)c3c(OC)cccc3o2)cc1OC</smiles><smiles>O=c1cc(-c2ccc(O)c(O)c2)oc2ccc(C(F)(F)F)cc12</smiles>

Figure 1. Synthesis of 5,3',4'-trimethoxyflavone (6j) and 5,3',4'-trihydroxyflavone (7j). i) $\mathrm{Me}_{2} \mathrm{SO}_{4}, \mathrm{~K}_{2} \mathrm{CO}_{3}$, acetone, rt, 6h; ii) DCC, DMAP, 3,4dimethoxybenzoic acid (3f); iii) $\mathrm{KOH}$, pyridine, $100^{\circ} \mathrm{C}, 10 \mathrm{~min}$; iv) $20 \% \mathrm{H}_{2} \mathrm{SO}_{4} /$ acetic acid, $\left.100^{\circ} \mathrm{C}, 10 \mathrm{~min} ; \mathrm{v}\right) 1.0 \mathrm{M} \mathrm{BBr}_{3} / \mathrm{CH}_{2} \mathrm{Cl}_{2}, \mathrm{rt}, 12 \mathrm{~h}$.

Synthesis of 5,3',4'-trimethoxyflavone (6j) and 5,3',4'trihydroxyflavone (7j) (Figure 1). General procedure for synthesis of methoxyflavones (Figure 2, 6a-6k) and hydroxyflavones (Figure 2, 7a-7k). To a suspension of 2',6'-dihydroxyacetophenone (1b, $6.53 \mathrm{~g}$, $42.9 \mathrm{mmol})$ and $\mathrm{K}_{2} \mathrm{CO}_{3}(8.89 \mathrm{~g}, 64.4 \mathrm{mmol})$ in acetone $(50 \mathrm{ml})$, dimethylsulfate $(4.07 \mathrm{ml}, 42.9 \mathrm{mmol})$ was dropwisely added. After the reaction mixture was refluxed overnight, the organic solvent was removed under reduced pressure. The residue was dissolved in dichloromethane $(40 \mathrm{ml})$ and the organic solution was washed with deionized water. The concentrated residue of the organic phase was chromatographed over silica gel (hexane/ethyl acetate; $85: 15$ ) to afford 2'-hydroxy-6'-methoxyacetphenone (2b, $3.35 \mathrm{~g}, 47 \%)$.

To a suspension of dicyclohexylcarbodiimide $(1.00 \mathrm{~g}, 4.85 \mathrm{mmol})$ and $N, N$-dimethyl-4-aminopyridine $(107 \mathrm{mg}, 0.876 \mathrm{mmol})$ in dry dichloromethane $(12 \mathrm{ml}), \mathbf{2 b}(718 \mathrm{mg}, 4.32 \mathrm{mmol})$ and then $3,4-$ dimethoxybenzoic acid (3f, $977 \mathrm{mg}, 5.37 \mathrm{mmol}$ ) were added. The reaction mixture was stirred at room temperature for 20 hours. The mixture was filtered to remove dicyclohexylurea as white precipitate. The solvent of the filtrate was removed under reduced pressure, and the residue was chromatographed over silica gel [hexane/dichloromethane; $2: 8$ ] to afford 6'-methoxy-2'-(3,4-dimethoxybenzoyloxy) acetophenone (4j) as a white solid. For 4j: ${ }^{1} \mathrm{H}-\mathrm{NMR}(\mathrm{CDCl} 3) \delta 2.49$ (s, 3H), $3.84(\mathrm{~s}$, $3 \mathrm{H}), 3.86(\mathrm{~s}, 3 \mathrm{H}), 3.87(\mathrm{~s}, 3 \mathrm{H}), 6.87(\mathrm{~d}, 1 \mathrm{H}, J=8.8 \mathrm{~Hz}), 7.19$ (dd, $1 \mathrm{H}$, $J=7.7,1.5 \mathrm{~Hz}), 7.42(\mathrm{~d}, 1 \mathrm{H}, J=1.5 \mathrm{~Hz}), 7.71(\mathrm{dd}, 1 \mathrm{H}, J=8.8,2.2 \mathrm{~Hz})$, $7.81(\mathrm{~d}, 1 \mathrm{H}, J=7.7 \mathrm{~Hz}), 7.90(\mathrm{~d}, 1 \mathrm{H}, J=2.2 \mathrm{~Hz})$.

To a suspension of $\mathrm{KOH}(212 \mathrm{mg}, 3.79 \mathrm{mmol})$ in dry pyridine ( $3 \mathrm{ml}) \mathbf{4 j}$ (573 mg, $1.74 \mathrm{mmol}$ ) was added and the mixture was stirred at $100^{\circ} \mathrm{C}$ for $10 \mathrm{~min}$. After being cooled to room temperature, the reaction mixture was neutralized with acetic acid (approximately $3.0 \mathrm{ml})$. The mixture was added to ethanol $(3.0 \mathrm{ml})$ and deionized water $(3.0 \mathrm{ml})$. The resulting precipitate was filtered and washed with cold ethanol to give 1-(3,4-dimethoxyphenyl)-3-(2-hydroxy-4methoxyphenyl) propane-1,3-dione $(\mathbf{5 j}, 350 \mathrm{mg}$, yield from $\mathbf{4 j}$ : $61 \%)$. For 5j: ${ }^{1} \mathrm{H}-\mathrm{NMR}\left(\mathrm{CDCl}_{3}\right) \delta 3.81(\mathrm{~s}, 2 \mathrm{H}), 3.87(\mathrm{~s}, 3 \mathrm{H}), 3.89$ $(\mathrm{d}, 6 \mathrm{H}), 6.47(\mathrm{~m}, 2 \mathrm{H}), 6.69(\mathrm{~s}, 1 \mathrm{H}), 7.38(\mathrm{~d}, 1 \mathrm{H}, \mathrm{J}=8.8 \mathrm{~Hz}), 7.44(\mathrm{~d}$, $1 \mathrm{H}, J=2.5 \mathrm{~Hz}), 7.48(\mathrm{~d}, 1 \mathrm{H}, J=1.5 \mathrm{~Hz}), 7.68(\mathrm{~d}, 1 \mathrm{H}, J=8 \mathrm{~Hz})$.
To a solution of containing $\mathbf{5 j}$ (431 $\mathrm{mg}, 1.30 \mathrm{mmol})$ dissolved in acetic acid $(6 \mathrm{ml}) \mathrm{kept}$ at $100^{\circ} \mathrm{C}, 20 \% \mathrm{H}_{2} \mathrm{SO}_{4} /$ acetic acid $(1 \mathrm{ml})$ was added. The mixture was stirred at $100^{\circ} \mathrm{C}$ for $10 \mathrm{~min}$. After cooling to room temperature, deionized water was added to the mixture. The resulting precipitate was filtered and washed with water, and was then chromatographed over silica gel (hexane/dichloromethane; $2: 8$ ) to afford 7,3'-dimethoxyflavone (6j, $379 \mathrm{mg}$, yield from $\mathbf{5 j}$ : $93 \%)$.

To a solution containing $\mathbf{6 j}(300 \mathrm{mg}, 1.12 \mathrm{mmol})$ in dry dichloromethane kept at $0^{\circ} \mathrm{C}, 1.0 \mathrm{M} \mathrm{BBr} / \mathrm{CH}_{2} \mathrm{Cl}_{2}(3.9 \mathrm{ml}$, $3.9 \mathrm{mmol}$ ) was carefully added. After the mixture stirred at room temperature for $12 \mathrm{~h}, 7 \mathrm{ml}$ of ethanol were added and the organic solvent was removed under reduced pressure. To the residue $11 \mathrm{ml}$ of boiling $50 \%$ ethanol were added. After being cooled to room temperature, the resulting precipitate was filtered and washed with $50 \%$ ethanol. Recrystallization from ethanol afforded $7 \mathbf{j}$ as yellow needles (211 mg, 74\%).

By combination of 2'-hydroxyacetophenone derivatives, namely 2'-hydroxy- (1a), 2'-hydroxy-6'-methoxy- (1b), 2'-hydroxy-5'methoxy- (1c), and 2'-hydroxy-4'-methoxy-acetophenones (1d), and benzoic acid (2a) and its derivatives, namely 2-methoxy- (2b), 3methoxy- (2c), 4-methoxy- (2d), 3,4,5-trimethoxy- (2e), and 3,4dimethoxybenzoic acids (2f), 11 methoxylated and 10 hydroxylated flavones were synthesized. Spectral data for $\mathbf{6 j}$ and $\mathbf{7 j}$ are shown in Table I along with data for other synthetic flavones.

Cell proliferation assay. HL60 cells were maintained in RPMI1640 medium supplemented with $10 \%$ fetal bovine serum. The level of cellular proliferation for HL60 cells grown in a 96-well microplate was measured by using Alamar Blue (Life Technologies Ltd., Tokyo, Japan). To each well, $100 \mu$ l of HL60 cell suspension $\left(1.0 \times 10^{4}\right.$ cells $\left./ 100 \mu \mathrm{l}\right)$ was were inoculated and $100 \mu \mathrm{l}$ of medium containing serial dilutions of the samples to be assayed was were added. After three days of incubation, $20 \mu \mathrm{l}$ of alamar blue was aseptically added to each well, and cells were further incubated for approximately 20 hours. Cellular proliferation (as a percentage that 


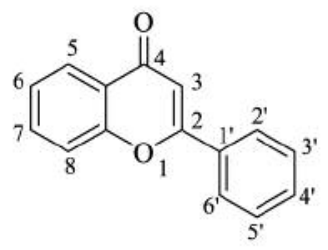

\begin{tabular}{|c|c|c|c|c|c|c|c|c|c|}
\hline \multirow{2}{*}{ Compound } & \multicolumn{7}{|c|}{ Substituents } & \multirow{2}{*}{$\begin{array}{l}\mathrm{IC}_{50} \\
(\mu \mathrm{M})\end{array}$} & \multirow{2}{*}{$\log p$} \\
\hline & $\mathrm{C} 5$ & C6 & $\mathrm{C} 7$ & $\mathrm{C} 2$ ' & $\mathrm{C} 3$, & $\mathrm{C} 4$ ' & $\mathrm{C} 5$ & & \\
\hline $6 a$ & $\mathrm{OMe}$ & $\mathrm{H}$ & $\mathrm{H}$ & $\mathrm{H}$ & $\mathrm{H}$ & $\mathrm{H}$ & $\mathrm{H}$ & 48 & 2.95 \\
\hline $6 b$ & $\mathrm{H}$ & $\mathrm{OMe}$ & $\mathrm{H}$ & $\mathrm{H}$ & $\mathrm{H}$ & $\mathrm{H}$ & $\mathrm{H}$ & $400<$ & 2.95 \\
\hline $6 c$ & $\mathrm{H}$ & $\mathrm{H}$ & $\mathrm{OMe}$ & $\mathrm{H}$ & $\mathrm{H}$ & $\mathrm{H}$ & $\mathrm{H}$ & 68 & 2.95 \\
\hline $6 d$ & $\mathrm{H}$ & $\mathrm{H}$ & $\mathrm{H}$ & $\mathrm{OMe}$ & $\mathrm{H}$ & $\mathrm{H}$ & $\mathrm{H}$ & 101 & 2.95 \\
\hline $6 e$ & $\mathrm{H}$ & $\mathrm{H}$ & $\mathrm{H}$ & $\mathrm{H}$ & $\mathrm{OMe}$ & $\mathrm{H}$ & $\mathrm{H}$ & 206 & 2.95 \\
\hline $6 f$ & $\mathrm{H}$ & $\mathrm{H}$ & $\mathrm{H}$ & $\mathrm{H}$ & $\mathrm{H}$ & $\mathrm{OMe}$ & $\mathrm{H}$ & 77 & 2.95 \\
\hline $6 \mathrm{~g}$ & $\mathrm{H}$ & $\mathrm{H}$ & $\mathrm{H}$ & $\mathrm{H}$ & $\mathrm{OMe}$ & $\mathrm{OMe}$ & $\mathrm{OMe}$ & $400<$ & 2.69 \\
\hline $6 h$ & $\mathrm{OMe}$ & $\mathrm{H}$ & $\mathrm{H}$ & $\mathrm{H}$ & $\mathrm{OMe}$ & $\mathrm{H}$ & $\mathrm{H}$ & 46 & 2.82 \\
\hline $6 i$ & $\mathrm{OMe}$ & $\mathrm{H}$ & $\mathrm{H}$ & $\mathrm{H}$ & $\mathrm{H}$ & $\mathrm{OMe}$ & $\mathrm{H}$ & 36 & 2.82 \\
\hline $6 j$ & $\mathrm{OMe}$ & $\mathrm{H}$ & $\mathrm{H}$ & $\mathrm{H}$ & $\mathrm{OMe}$ & $\mathrm{OMe}$ & $\mathrm{H}$ & $400<$ & 2.69 \\
\hline $6 \mathrm{k}$ & $\mathrm{H}$ & $\mathrm{OMe}$ & $\mathrm{H}$ & $\mathrm{H}$ & $\mathrm{H}$ & $\mathrm{OMe}$ & $\mathrm{H}$ & $400<$ & 2.82 \\
\hline 8 & $\mathrm{H}$ & $\mathrm{H}$ & $\mathrm{H}$ & $\mathrm{H}$ & $\mathrm{OMe}$ & $\mathrm{OMe}$ & $\mathrm{H}$ & $400<$ & 2.82 \\
\hline $7 \mathbf{a}$ & $\mathrm{OH}$ & $\mathrm{H}$ & $\mathrm{H}$ & $\mathrm{H}$ & $\mathrm{H}$ & $\mathrm{H}$ & $\mathrm{H}$ & $400<$ & 2.68 \\
\hline $7 \mathbf{b}$ & $\mathrm{H}$ & $\mathrm{OH}$ & $\mathrm{H}$ & $\mathrm{H}$ & $\mathrm{H}$ & $\mathrm{H}$ & $\mathrm{H}$ & 89 & 2.68 \\
\hline $7 \mathrm{c}$ & $\mathrm{H}$ & $\mathrm{H}$ & $\mathrm{OH}$ & $\mathrm{H}$ & $\mathrm{H}$ & $\mathrm{H}$ & $\mathrm{H}$ & $400<$ & 2.68 \\
\hline $7 d$ & $\mathrm{H}$ & $\mathrm{H}$ & $\mathrm{H}$ & $\mathrm{OH}$ & $\mathrm{H}$ & $\mathrm{H}$ & $\mathrm{H}$ & $400<$ & 2.68 \\
\hline $7 e$ & $\mathrm{H}$ & $\mathrm{H}$ & $\mathrm{H}$ & $\mathrm{H}$ & $\mathrm{OH}$ & $\mathrm{H}$ & $\mathrm{H}$ & $400<$ & 2.68 \\
\hline $7 f$ & $\mathrm{H}$ & $\mathrm{H}$ & $\mathrm{H}$ & $\mathrm{H}$ & $\mathrm{H}$ & $\mathrm{OH}$ & $\mathrm{H}$ & $400<$ & 2.68 \\
\hline $7 \mathrm{~g}$ & $\mathrm{H}$ & $\mathrm{H}$ & $\mathrm{H}$ & $\mathrm{H}$ & $\mathrm{OH}$ & $\mathrm{OH}$ & $\mathrm{OH}$ & 196 & 1.9 \\
\hline $7 \mathrm{~h}$ & $\mathrm{OH}$ & $\mathrm{H}$ & $\mathrm{H}$ & $\mathrm{H}$ & $\mathrm{OH}$ & $\mathrm{H}$ & $\mathrm{H}$ & $400<$ & 2.29 \\
\hline $7 i$ & $\mathrm{OH}$ & $\mathrm{H}$ & $\mathrm{H}$ & $\mathrm{H}$ & $\mathrm{H}$ & $\mathrm{OH}$ & $\mathrm{H}$ & 28 & 2.29 \\
\hline $7 \mathbf{j}$ & $\mathrm{OH}$ & $\mathrm{H}$ & $\mathrm{H}$ & $\mathrm{H}$ & $\mathrm{OH}$ & $\mathrm{OH}$ & $\mathrm{H}$ & 13 & 1.9 \\
\hline $7 \mathrm{k}$ & $\mathrm{H}$ & $\mathrm{OH}$ & $\mathrm{H}$ & $\mathrm{H}$ & $\mathrm{H}$ & $\mathrm{OH}$ & $\mathrm{H}$ & n.t. & 2.29 \\
\hline 9 & $\mathrm{H}$ & $\mathrm{H}$ & $\mathrm{H}$ & $\mathrm{H}$ & $\mathrm{OH}$ & $\mathrm{OH}$ & $\mathrm{H}$ & 51 & 2.29 \\
\hline 10 & $\mathrm{H}$ & $\mathrm{H}$ & $\mathrm{H}$ & $\mathrm{H}$ & $\mathrm{H}$ & $\mathrm{H}$ & $\mathrm{H}$ & 64 & 3.07 \\
\hline
\end{tabular}

Figure 2. Structures, $I C_{50}(\mu M)$ and log P of methoxyflavones and hydroxyflavones. Calculation of log $p$ was done by ChemDraw Professionl 15.1 (Perkin-Elmer Infomatics, Inc.).

of the untreated control) was calculated with the following equation: Proliferation (\%)

$$
\begin{aligned}
& \text { ([ }\left(A_{570}-A_{595}\right) \text { of test agent dilution]- } \\
& \text { [( } \left.\left.A_{570}-A_{595}\right) \text { of blank] }\right) \\
& =\frac{\left(\left[\left(A_{570}-A_{595}\right) \text { of positive growth control }\right]-\right.}{\left.\left[\left(A_{570}-A_{595}\right) \text { of blank }\right]\right)}
\end{aligned}
$$

where $A_{570}$ and $A_{595}$ are the absorbance at $570 \mathrm{~nm}$ and $595 \mathrm{~nm}$, respectively.

\section{Results and Discussion}

Chemistry. In this study, 11 methylated flavones and 10 hydroxylated flavones were synthesized. The synthetic route to 5,3 ', 4' -trihydroxyflavone (7j) through 5,3',4'-trimethoxyflavone $(\mathbf{6 j})$ is illustrated in Figure 1. 2',6'-Dihydroxyacetophenone (1b) was first treated with dimethylsulfate and $\mathrm{K}_{2} \mathrm{CO}_{3}$ in dry acetone to afford 2'-hydroxy-6'methoxyactophenone (2b). In spite of the increased ratio of 
Table I. Analytical data of synthesized compounds.

Compound
weight (yield)

weight (yield ${ }^{1} \mathrm{H}-\mathrm{NMR}$

${ }^{1} \mathrm{H}-\mathrm{NMR}$

Compound weight (yield) ${ }^{1} \mathrm{H}-\mathrm{NMR}$

Compound weight (yield) ${ }^{1} \mathrm{H}-\mathrm{NMR}$

Compound weight (yield) ${ }^{1} \mathrm{H}-\mathrm{NMR}$

Compound weight (yield) ${ }^{1} \mathrm{H}-\mathrm{NMR}$
5-Methoxyflavone (6a) $490 \mathrm{mg}(42 \%)$ $4.00(\mathrm{~s}, 3 \mathrm{H}), 6.75(\mathrm{~s}, 1 \mathrm{H})$, $6.82(\mathrm{dd}, 1 \mathrm{H}, \mathrm{J}=8,1 \mathrm{~Hz})$,

$7.13(\mathrm{dd}, 1 \mathrm{H}, \mathrm{J}=8,1 \mathrm{~Hz}), 7.51$ $(\mathrm{m}, 3 \mathrm{H}), 7.60(\mathrm{t}, 1 \mathrm{H}$, $\mathrm{J}=8 \mathrm{~Hz}), 7.89(\mathrm{~m}, 2 \mathrm{H})$.

2'-Methoxyflavone (6d) $2.20 \mathrm{~g}(91 \%)$ 3.93 (s, 3H), 7.04 (d, 1H, J=8.4 Hz), $7.11(\mathrm{dt}, 1 \mathrm{H}, \mathrm{J}=6.6,1.1 \mathrm{~Hz})$ $7.15(\mathrm{~s}, 1 \mathrm{H}), 7.40(\mathrm{dt}$, $1 \mathrm{H}, \mathrm{J}=5.8,1.1 \mathrm{~Hz})$, $7.48(\mathrm{dt}, 1 \mathrm{H}, \mathrm{J}=5.5,1.8 \mathrm{~Hz})$ $7.52(\mathrm{dd}, 1 \mathrm{H}, \mathrm{J}=7.7,0.7 \mathrm{~Hz})$, $7.67(\mathrm{dt}, 1 \mathrm{H}, \mathrm{J}=5.5,1.8 \mathrm{~Hz})$, $7.90(\mathrm{dd}, 1 \mathrm{H}, \mathrm{J}=6.2,1.8 \mathrm{~Hz})$, $8.23(\mathrm{dd}, 1 \mathrm{H}, \mathrm{J}=6.2,1.8 \mathrm{~Hz})$

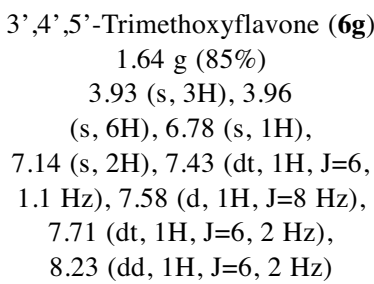

5,3',4'-Trimethoxyflavone (6j) $955 \mathrm{mg}(71 \%)$

3.96 (s, 3H), 3.97 (s, 3H), 4.00 (s, 3H), $6.80(\mathrm{~s}, 1 \mathrm{H}), 6.83(\mathrm{~d}$,

$1 \mathrm{H}, \mathrm{J}=8.4 \mathrm{~Hz}), 6.97(\mathrm{~d}, 1 \mathrm{H}$ $\mathrm{J}=8.4 \mathrm{~Hz}), 7.14(\mathrm{~d}, 1 \mathrm{H}, \mathrm{J}=8.8 \mathrm{~Hz})$, $7.35(\mathrm{~d}, 1 \mathrm{H}, \mathrm{J}=2.2 \mathrm{~Hz}), 7.55(\mathrm{dd}$, $1 \mathrm{H}, \mathrm{J}=6.6,1.8 \mathrm{~Hz})$, $7.59(\mathrm{~d}, 1 \mathrm{H}, \mathrm{J}=8.4 \mathrm{~Hz})$.

6-Hydroxyflavone (7b) $352 \mathrm{mg}(62 \%)$ $6.85(\mathrm{~s}, 1 \mathrm{H}), 7.28(\mathrm{dd}, 1 \mathrm{H}$, $\mathrm{J}=5.8,2.9 \mathrm{~Hz}), 7.42(\mathrm{~d}, 1 \mathrm{H}, \mathrm{J}=2.9)$, $7.56(\mathrm{~m}, 3 \mathrm{H}), 7.60(\mathrm{~d}, 1 \mathrm{H}$, $\mathrm{J}=8.8 \mathrm{~Hz}), 8.02(\mathrm{dt}, 2 \mathrm{H}$, $\mathrm{J}=5.5,2.5 \mathrm{~Hz}$ ), 9.6 (br.s, $1 \mathrm{H})$

Compound weight (yield) ${ }^{1} \mathrm{H}-\mathrm{NMR}$
3'-Hydroxyflavone (7e) $473 \mathrm{mg}(77 \%)$ $6.86(\mathrm{~s}, 1 \mathrm{H}), 7.00(\mathrm{dt}, 1 \mathrm{H}$, $\mathrm{J}=5.2,1.4 \mathrm{~Hz}), 7.37(\mathrm{t}, 1 \mathrm{H}$, $\mathrm{J}=8.1 \mathrm{~Hz}), 7.43(\mathrm{t}, 1 \mathrm{H}, \mathrm{J}=1.8 \mathrm{~Hz})$, $7.50(\mathrm{~m}, 2 \mathrm{H}), 7.72(\mathrm{dd}, 1 \mathrm{H}$, $\mathrm{J}=7.7,0.7 \mathrm{~Hz}), 7.82(\mathrm{dt}$

$1 \mathrm{H}, \mathrm{J}=5.2,1.8 \mathrm{~Hz}), 8.14(\mathrm{dd}, 1 \mathrm{H}$, $\mathrm{J}=6.6,1.1 \mathrm{~Hz}$ ), 9.5 (br.s, 1H)
6-Methoxyflavone (6b) $1.05 \mathrm{~g}(72 \%)$

$3.91(\mathrm{~s}, 3 \mathrm{H}), 6.95(\mathrm{~s}, 1 \mathrm{H})$

$7.31(\mathrm{dd}, 1 \mathrm{H}, \mathrm{J}=7.1,3 \mathrm{~Hz})$ $7.53(\mathrm{~m}, 4 \mathrm{H}), 7.59(\mathrm{~d}, 1 \mathrm{H}, \mathrm{J}=3 \mathrm{~Hz})$, $7.93(\mathrm{~m}, 2 \mathrm{H})$

3'-Methoxyflavone (6e) $1.21 \mathrm{~g}(75 \%)$

$3.89(\mathrm{~s}, 3 \mathrm{H}), 6.85(\mathrm{~s}, 1 \mathrm{H}), 7.08$

$(\mathrm{dt}, 1 \mathrm{H}, \mathrm{J}=5.5,2.6 \mathrm{~Hz})$, $7.44(\mathrm{~m}, 3 \mathrm{H})$,

$7.51(\mathrm{dt}, 1 \mathrm{H}, \mathrm{J}=6.2,0.7 \mathrm{~Hz})$, $7.57(\mathrm{~d}, 1 \mathrm{H}, \mathrm{J}=8.4 \mathrm{~Hz})$, $7.71(\mathrm{dt}, 1 \mathrm{H}, \mathrm{J}=5.5$, $1.8 \mathrm{~Hz}), 8.23$ (dd, $1 \mathrm{H}, \mathrm{J}=6.2,1.8 \mathrm{~Hz}$ )

\section{5,3'-Dimethoxyflavone (6h) $659 \mathrm{mg}(65 \%)$}

3.90 (s, 3H), 3.92 (s, 3H), 6.70 $(\mathrm{s}, 1 \mathrm{H}), 6.98(\mathrm{~d}, 1 \mathrm{H}, \mathrm{J}=8.0 \mathrm{~Hz})$, $7.14(\mathrm{dd}, 1 \mathrm{H}, \mathrm{J}=5.9,1.8 \mathrm{~Hz})$,

$7.24(\mathrm{dd}, 1 \mathrm{H}, \mathrm{J}=7.4,1.1 \mathrm{~Hz})$,

$7.48(\mathrm{t}, 1 \mathrm{H}, \mathrm{J}=7.7 \mathrm{~Hz}), 7.56$

$(\mathrm{t}, 1 \mathrm{H}, \mathrm{J}=1.8 \mathrm{~Hz}), 7.61$

(dt, 1H, J=5.1, 1.8 Hz),

$7.67(\mathrm{t}, 1 \mathrm{H}, \mathrm{J}=8.4 \mathrm{~Hz})$

6,4'-Dimethoxyflavone (6k) $674 \mathrm{mg}(74 \%)$

$3.86(\mathrm{~s}, 3 \mathrm{H}), 3.87(\mathrm{~s}, 3 \mathrm{H}), 6.95$ $(\mathrm{s}, 1 \mathrm{H}), 7.12(\mathrm{~d}, 2 \mathrm{H}, \mathrm{J}=8.8 \mathrm{~Hz})$, $7.41(\mathrm{~d}, 1 \mathrm{H}, \mathrm{J}=3.3 \mathrm{~Hz}), 7.43$ (d, 1H, J=2.2 Hz), 7.75 (dd,

$1 \mathrm{H}, \mathrm{J}=4.4,2.6 \mathrm{~Hz}$ ), 8.07 (dd, $2 \mathrm{H}, \mathrm{J}=5.1,1.8 \mathrm{~Hz}$ ).

\section{7-Hydroxyflavone (7c)} $434 \mathrm{mg}(38 \%)$

$6.82(\mathrm{~s}, 1 \mathrm{H}), 6.99(\mathrm{dd}, 1 \mathrm{H}$ $\mathrm{J}=7.0,1.1 \mathrm{~Hz}), 7.04(\mathrm{~d}, 1 \mathrm{H}$, $\mathrm{J}=1.1 \mathrm{~Hz}), 7.38(\mathrm{~m}, 2 \mathrm{H})$, $7.49(\mathrm{~m}, 3 \mathrm{H}), 7.70(\mathrm{~d}$, 1H, J=7.0 Hz), 9.3 (br.s, 1H)

4'-Hydroxyflavone (7f) $3.90 \mathrm{~g}(83 \%)$

$6.87(\mathrm{~d}, 1 \mathrm{H}, \mathrm{J}=4.4 \mathrm{~Hz}), 6.95(\mathrm{~m}, 2 \mathrm{H})$, $7.49(\mathrm{dd}, 1 \mathrm{H}, \mathrm{J}=6.9,0.7 \mathrm{~Hz})$, $7.79(\mathrm{~m}, 2 \mathrm{H}), 7.97(\mathrm{~m}, 2 \mathrm{H})$, $8.04(\mathrm{dd}, 1 \mathrm{H}, \mathrm{J}=6.9,0.8 \mathrm{~Hz})$, 10.3 (br.s, $1 \mathrm{H}$ )

\section{7-Methoxyflavone (6c)}

$2.58 \mathrm{~g}(71 \%)$

$3.73(\mathrm{~s}, 3 \mathrm{H}), 7.07$ (dd, 1H, J=7.1, $3 \mathrm{~Hz})$, $7.15(\mathrm{~s}, 1 \mathrm{H}), 7.21(\mathrm{~d}, 1 \mathrm{H}, \mathrm{J}=7.1 \mathrm{~Hz})$, $7.72(\mathrm{~m}, 3 \mathrm{H}), 7.59(\mathrm{~d}, 1 \mathrm{H}, \mathrm{J}=3 \mathrm{~Hz})$, $8.01(\mathrm{~m}, 2 \mathrm{H})$.

4'-Methoxyflavone (6f) $176 \mathrm{mg}(78 \%)$ 3.87 (s, 3H), $6.81(\mathrm{~s}, 1 \mathrm{H}), 7.02$ $(\mathrm{dd}, 1 \mathrm{H}, \mathrm{J}=7.1,1.5 \mathrm{~Hz}), 7.41(\mathrm{dt}$, $1 \mathrm{H}, \mathrm{J}=7.3,1.0 \mathrm{~Hz}), 7.55(\mathrm{~d}, 1 \mathrm{H}$, $\mathrm{J}=8.4 \mathrm{~Hz}), 7.69(\mathrm{dt}, 1 \mathrm{H}, \mathrm{J}=8.1$, $1.5 \mathrm{~Hz}), 7.89(\mathrm{~d}, 2 \mathrm{H}, \mathrm{J}=7 \mathrm{~Hz})$, $8.22(\mathrm{~d}, 2 \mathrm{H}, \mathrm{J}=7 \mathrm{~Hz})$.

\section{5,4'-Dimethoxyflavone (6i) $779 \mathrm{mg}(69 \%)$}

3.87 (s, 3H), 3.99 (s, 3H), 6.67 $(\mathrm{s}, 1 \mathrm{H}), 6.81(\mathrm{~d}, 1 \mathrm{H}, \mathrm{J}=8.1 \mathrm{~Hz})$, $6.99(\mathrm{dd}, 2 \mathrm{H}, \mathrm{J}=4.8,2.2 \mathrm{~Hz})$,

$7.11(\mathrm{dd}, 1 \mathrm{H}, \mathrm{J}=7.7,0.7 \mathrm{~Hz})$, $7.55(\mathrm{t}, 1 \mathrm{H}, \mathrm{J}=8.4 \mathrm{~Hz})$,

$7.84(\mathrm{dd}, 2 \mathrm{H}, \mathrm{J}=4.8,1.8 \mathrm{~Hz})$

5-Hydroxyflavone (7a) $211 \mathrm{mg}(74 \%)$

$6.79(\mathrm{dt}, 1 \mathrm{H}, \mathrm{J}=8,1.2 \mathrm{~Hz})$ $6.92(\mathrm{~s}, 1 \mathrm{H}), 7.16(\mathrm{t}, 1 \mathrm{H}$, $\mathrm{J}=8.0 \mathrm{~Hz}), 7.63(\mathrm{~m}, 3 \mathrm{H})$, $7.68(\mathrm{dt}, 1 \mathrm{H}, \mathrm{J}=8,1.2 \mathrm{~Hz})$, $8.11(\mathrm{dd}, 2 \mathrm{H}, \mathrm{J}=6.2,1.4 \mathrm{~Hz})$, 12.6 (br.s, $1 \mathrm{H}$ )

2'-Hydroxyflavone (7d) $863 \mathrm{mg}(91 \%)$ $6.99(\mathrm{dt}, 1 \mathrm{H}, \mathrm{J}=7.0,1.1 \mathrm{~Hz})$,

$7.04(\mathrm{~d}, 1 \mathrm{H}, \mathrm{J}=1.1 \mathrm{~Hz})$, $7.38(\mathrm{~m}, 2 \mathrm{H}), 7.49(\mathrm{dt}, 1 \mathrm{H}$, $\mathrm{J}=7.0,1.1 \mathrm{~Hz}), 7.70(\mathrm{~d}, 1 \mathrm{H}$, $\mathrm{J}=8.4 \mathrm{~Hz}), 7.80(\mathrm{dt}, 1 \mathrm{H}, \mathrm{J}=1.4 \mathrm{~Hz})$, $7.96(\mathrm{dd}, 1 \mathrm{H}, \mathrm{J}=6.2,1.8 \mathrm{~Hz})$, $8.14(\mathrm{dd}, 1 \mathrm{H}, \mathrm{J}=6.2,1.8 \mathrm{~Hz})$, 9.4 (br.s, 1H)

3',4',5'-Trihydroxyflavone (7g) $530 \mathrm{mg}(65 \%)$

$6.63(\mathrm{~s}, 1 \mathrm{H}), 7.01(\mathrm{~m}, 2 \mathrm{H})$, $7.49(\mathrm{dt}, 1 \mathrm{H}, \mathrm{J}=7.0,1.1 \mathrm{~Hz})$,

$7.69(\mathrm{~d}, 1 \mathrm{H}, \mathrm{J}=8.1 \mathrm{~Hz})$ $7.81(\mathrm{dt}, 1 \mathrm{H}, \mathrm{J}=5.2,1.8 \mathrm{~Hz})$, $8.03(\mathrm{dd}, 1 \mathrm{H}, \mathrm{J}=6.2,1.5 \mathrm{~Hz})$, 9.10 (br.s, 1H), 9.41 (br.s, 2H). 
Compound weight (yield) ${ }^{1} \mathrm{H}-\mathrm{NMR}$

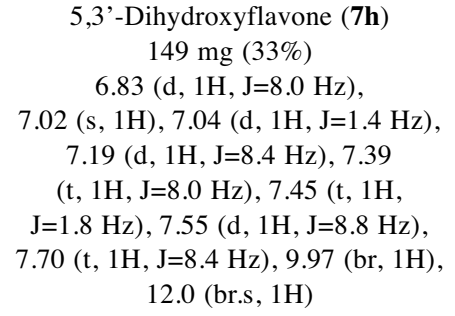

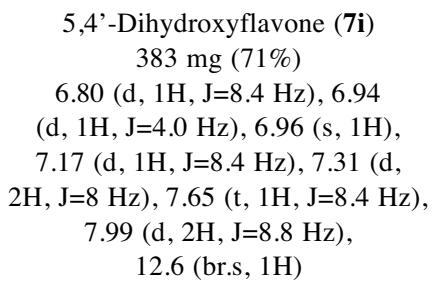

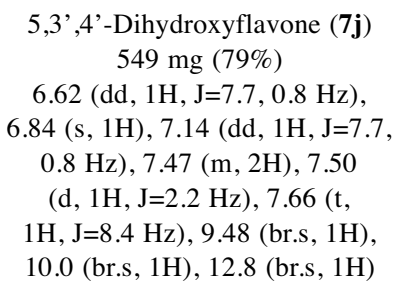

iodomethane added, 2',6'-dimethoxyacetophenone was not produced, fortunately because the nucleophilicity of either of two ortho-hydroxyl groups was weakened by the adjacent acetyl carbonyl group.

2b was then reacted with 3,4-dimethoxybenzoic acid (3f)

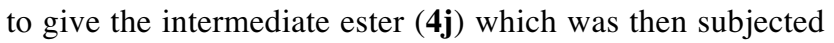
to the Baker-Venkataraman rearrangement in dry pyridine in the presence of $\mathrm{KOH}$, to afford the 1,3-dione (5j). Acidcatalyzed cyclization of $\mathbf{5} \mathbf{j}$ in acetic acid in the presence of $\mathrm{H}_{2} \mathrm{SO}_{4}$ yielded 5,3',4'-trimethoxyflavones (6j) in yields of $25-35 \%$, which was then demethylated using $\mathrm{BBr} 3$ to give $5,3^{\prime}, 4$ '-trihydroxyflavones $(\mathbf{7 j})$.

Antiproliferative activity. These synthesized compounds were tested for their antiproliferative activity in vitro against HL60 leukemic cells using serial dilution method in 96-well microplates and flavone (10) was used as the positive control. The obtained results are summarized in Figure 2. Among the compounds tested, 5,3',4'trihydoxyflavone $\left(\mathbf{7} \mathbf{j}, \mathrm{IC}_{50}=13 \mu \mathrm{M}\right)$ demonstrated the most potent activity, followed by 5,4'-dihydroxyflavone (7i, $\left.\mathrm{IC}_{50}=28 \mu \mathrm{M}\right)$. Furthermore the 5-methoxylated derivatives, namely 5,4'-dimethoxyflavone (6i, $\left.\mathrm{IC}_{50}=36 \mu \mathrm{M}\right), 5,3$ 'dimethoxyflavone $\left(6 \mathbf{h}, \quad \mathrm{IC}_{50}=46 \mu \mathrm{M}\right)$ and 5methoxyflavone $\left(6 \mathbf{a}, \mathrm{IC}_{50}=48 \mu \mathrm{M}\right)$ also demonstrated significant activity.

Structure and activity relationship. The introduction of hydroxyl groups both at $\mathrm{C} 3$ ' and $\mathrm{C} 4$ ' positions in flavone (10, $\left.\mathrm{IC}_{50}=64 \mu \mathrm{M}\right)$, slightly improved the activity (9, $\left.\mathrm{IC}_{50}=51 \mu \mathrm{M}\right)$. The role of 3',4'-dihydroxy moiety for in the activity was also supported by the observation that methylation of hydroxyl groups at C3' and C4' drastically reduced the activity $\left(8, \mathrm{IC}_{50}>400 \mu \mathrm{M}\right)$. The results that the 3',4'-dihydroxy moiety of dihydroxyflavone independently showed the antiproliferative activity, gave us precise information about the role of the this structural moiety, although the importance of 3',4'-dihydroxy moiety in the polyhydroxyflavones had been reported $(20,21)$. Among the dihydroxyflavones tested, namely compounds $\mathbf{7 h}, \mathbf{7 i}, \mathbf{7 k}$ and 9, the most potent activity was shown by 5,4'dihydroxyflavone $\left(\mathbf{7 i}, \mathrm{IC}_{50}=28 \mu \mathrm{M}\right)$ which does not have 3',4'-dihydroxy moiety. Taking these observations into account, the 3',4'-dihydroxy and 5,4'-dihydroxy moieties were suggested to have a different mechanism of action. The activity enhancements found in $7 \mathbf{j}\left(\mathrm{IC}_{50}=13 \mu \mathrm{M}\right)$ both from 4-hydroxylation of $9 \quad\left(\mathrm{IC}_{50}=51 \mu \mathrm{M}\right)$ and from 3'-hydroxylation of $7 \mathbf{i} \quad\left(\mathrm{IC}_{50}=28 \mu \mathrm{M}\right)$ indicated the synergistic effects of these two dihydroxy moieties and thus strongly supported the above suggestion.

A noteworthy reduction of the antiproliferative activity was observed for compounds $\mathbf{6 j}$ and 8 bearing 3',4'-dimethoxyl group. Moreover, the removal of one or two methoxyl groups from the B-ring of compound $\mathbf{6 j}\left(\mathrm{IC}_{50}>400 \mu \mathrm{M}\right)$ caused an increased potency of compounds $6 \mathbf{a}\left(\mathrm{IC}_{50}=48 \mu \mathrm{M}\right), \mathbf{6 h}$ $\left(\mathrm{IC}_{50}=46 \mu \mathrm{M}\right)$ and $6 \mathbf{i}\left(\mathrm{IC}_{50}=36 \mu \mathrm{M}\right)$. These findings indicating that the increase of methoxyl groups on the B-ring decrease activity are consistent with our previous study where the attachment of more than two methoxyl groups on the B-ring completely diminished the activity when the number of A-ring methoxyl groups was 0-3 (19).

The position of methoxyl group on A-ring had significant effects on the activity; 5-methoxyflavone (6a, $\left.\mathrm{IC}_{50}=48 \mu \mathrm{M}\right)$ and 7-methoxyflavone $\left(6 \mathbf{c}, \mathrm{IC}_{50}=68 \mu \mathrm{M}\right)$ displayed the moderate activity whereas 6-methoxyflavone $\left(6 \mathbf{b}, \mathrm{IC}_{50}>400 \mu \mathrm{M}\right)$ had no activity. Contrarily, the intact hydroxyl group on A-ring had an opposite effects on the activity profile; only 6-hydroxyflavone $\left(7 \mathbf{b}, \mathrm{IC}_{50}=89 \mu \mathrm{M}\right)$ showed the activity among the A-ring monohydroxylated derivatives. These findings suggested the important role of the A-ring in the interaction with target molecules.

In summary the data presented here demonstrated that the mechanisms of action of the 3',4'-dihydroxy and 5,4'dihydroxy moieties were different and thus synergistic, and that the increased number of B-ring methoxyl groups reduced the activity of PMFs towards HL60 cells. 


\section{References}

1 Pasman WJ, van Erk MJ, Klöpping WAA, Pellis L, Wopereis S, Bijlsma S, Hendriks HFJ and Kardinaal AFM: Nutrigenomics approach elucidates health-promoting effects of high vegetable intake in lean and obese men. Genes Nutr 8: 507-521, 2013.

$2 \mathrm{Li} \mathrm{C}$ and Schluesener H: Health-promoting effects of the citrus flavanone hesperidin. Crit Rev Food Sci Nutr 57: 613-631, 2017.

3 Silva BJ, Seca AM, Barreto Mdo C and Pinto DC: Recent Breakthroughs in the Antioxidant and Anti-Inflammatory Effects of Morella and Myrica Species. Int J Mol Sci 28: 17160-17180, 2015.

4 Manthey JA, Grohmann K and Guthrie N: Biological properties of citrus flavonoids pertaining to cancer and inflammation. Curr Med Chem 8: 135-153, 2001.

5 Elsharkawy ER: Isolation of phytoconstituents and evaluation of anticancer and Antioxidant potential of Launaea mucronata (Forssk.) Muschl. subsp. Pak J Pharm Sci 30: 399-405, 2017.

6 Johnson JL and de Mejia EG: Flavonoid apigenin modified gene expression associated with inflammation and cancer and induced apoptosis in human pancreatic cancer cells through inhibition of GSK-3 $\beta /$ NF-kB signaling cascade. Mol Nutr Food Res 57: 21122127, 2013.

7 Romagnolo DF and Selmin OI: Flavonoids and cancer prevention: a review of the evidence. J Nutr Gerontol Geriatr 31: 206-238, 2012.

8 Kawaii S, Endo K, Tokiwano T and Yoshizawa Y: Relationship between structure and antiproliferative activity of 1azaflavanones. Anticancer Res 32: 2819-2825, 2012.

9 Forbes AM, Lin H, Meadows GG and Meier GP: Synthesis and anticancer activity of new flavonoid analogs and inconsistencies in assays related to proliferation and viability measurements. Int J Oncol 45: 831-842, 2014.

10 Whitman SC, Kurowska EM, Manthey JA and Daugherty A: Nobiletin, a citrus flavonoid isolated from tangerines, selectively inhibits class A scavenger receptor-mediated metabolism of acetylated LDL by mouse macrophages. Atherosclerosis 178: 2532, 2005.

11 Yoshimizu N, Otani Y, Saikawa Y, Kubota T, Yoshida M, Furukawa T, Kumai K, Kameyama K, Fujii M, Yano M, Sato T, Ito $\mathrm{T}$ and Kitajima M: Anti-tumor effects of nobiletin, a citrus flavonoid, on gastric cancer include: antiproliferative effects, induction of apoptosis and cell cycle deregulation. Aliment Pharmacol Ther 20: 95-101, 2004.

12 Lin N, Sato T, Takayama Y, Mimaki Y, Sashida Y, Yano M and Ito A: Novel anti-inflammatory actions of nobiletin, a citrus polymethoxy flavonoid, on human synovial fibroblasts and mouse macrophages. Biochem Pharmacol 65: 2065-2071, 2003.
13 Guo S, Qiu P, Xu G, Wu X, Dong P, Yang G, Zheng J, McClements DJ and Xiao H: Synergistic anti-inflammatory effects of nobiletin and sulforaphane in lipopolysaccharide-stimulated RAW 264.7 cells. J Agric Food Chem 60: 2157-2164, 2012.

14 Al Rahim M, Nakajima A, Saigusa D, Tetsu N, Maruyama Y, Shibuya M, Yamakoshi H, Tomioka Y, Iwabuchi Y, Ohizumi Y and Yamakuni T: 4'-Demethylnobiletin, a bioactive metabolite of nobiletin enhancing PKA/ERK/CREB signaling, rescues learning impairment associated with NMDA receptor antagonism via stimulation of the ERK cascade. Biochemistry 48: 7713-7721, 2009.

15 Li S, Pan MH, Lai CS, Lo CY, Dushenkov S and Ho CT: Isolation and syntheses of polymethoxyflavones and hydroxylated polymethoxyflavones as inhibitors of HL-60 cell lines. Bioorg Med Chem 15: 3381-3389, 2007.

16 Kawaii S, Tomono Y, Katase E, Ogawa K and Yano M: HL-60 differentiating activity and flavonoid content of the readily extractable fraction prepared from citrus juices. J Agric Food Chem 47: 128-135, 1999.

17 Kawaii S, Tomono Y, Katase E, Ogawa $\mathrm{K}$ and Yano M: Quantitation of flavonoid constituents in citrus fruits. J Agric Food Chem 47: 3565-3571, 1999.

18 Kawaii S, Tomono Y, Katase E, Ogawa K, Nonomura-Nakano M, Nesumi H, Yoshida T, Sugiura M and Yano M: Quantitative study of fruit flavonoids in citrus hybrids of King (C. nobilis) and Mukaku Kishu (C. kinokuni). J Agric Food Chem 49: 39823986, 2001.

19 Kawaii S, Ikuina T, Hikima T, Tokiwano T and Yoshizawa Y: Relationship between structure and antiproliferative activity of polymethoxyflavones towards HL60 cells. Anticancer Res 32: 5239-5244, 2012.

20 Kawaii S, Tomono Y, Katase E, Ogawa $\mathrm{K}$ and Yano M: Antiproliferative activity of flavonoids on several cancer cell lines. Biosci Biotechnol Biochem 63: 896-899, 1999.

21 Grigalius I and Petrikaite V: Relationship between Antioxidant and Anticancer Activity of Trihydroxyflavones. Molecules 22: 2169,2017
Received August 30, 2018

Revised September 16, 2018 Accepted September 18, 2018 